University of Nebraska - Lincoln

DigitalCommons@University of Nebraska - Lincoln

February 1991

\title{
Substrate-induced magnetic ordering of rare-earth overlayers
}

Peter A. Dowben

University of Nebraska-Lincoln, pdowben@unl.edu

D. LaGraffe

Syracuse University

Dongqi Li

yracuse University, Syracuse, New York

A. Miller

Syracuse University

Ling Zhang

Syracuse University

See next page for additional authors

Follow this and additional works at: https://digitalcommons.unl.edu/physicsdowben

Part of the Physics Commons

Dowben, Peter A.; LaGraffe, D.; Li, Dongqi; Miller, A.; Zhang, Ling; Dottl, L.; and Onellion, M., "Substrateinduced magnetic ordering of rare-earth overlayers" (1991). Peter Dowben Publications. 64.

https://digitalcommons.unl.edu/physicsdowben/64

This Article is brought to you for free and open access by the Research Papers in Physics and Astronomy at DigitalCommons@University of Nebraska - Lincoln. It has been accepted for inclusion in Peter Dowben Publications by an authorized administrator of DigitalCommons@University of Nebraska - Lincoln. 


\section{Authors}

Peter A. Dowben, D. LaGraffe, Dongqi Li, A. Miller, Ling Zhang, L. Dottl, and M. Onellion 


\title{
Substrate-induced magnetic ordering of rare-earth overlayers
}

\author{
P. A. Dowben, ${ }^{*}$ D. LaGraffe, Dongqi Li, A. Miller, and Ling Zhang \\ Department of Physics, Syracuse University, Syracuse, New York 13244-1130 \\ L. Dottl and M. Onellion \\ University of Wisconsin, Madison, Wisconsin 53706 \\ (Received 1 August 1990)
}

\begin{abstract}
We have studied the magnetic ordering of terbium overlayers on $\mathrm{Cu}(100)$ and $\mathrm{Ni}(111)$, using angle-resolved photoemission. The $5 p_{3 / 2}$ to $5 p_{1 / 2}$ shallow-core-level branching ratios in different photoemission geometries provide a measure of the magnetic ordering in rare-earth-metal overlayers as a result of final-state effects in photoemission. We find that ferromagnetic substrates order paramagnetic terbium overlayers. This induced magnetic ordering is not a crystal-field effect and can be modeled by Ginzburg-Landau theory. Application of Ginzburg-Landau theory to our results suggests that the correlation length of paramagnetic terbium $\kappa^{-1}$ is between 2.5 and $3.5 \AA$. Reversible increases in the extent of magnetic ordering at temperatures below the $\mathrm{Tb}$ Curie temperature are observed for terbium overlayers on both $\mathrm{Cu}(100)$ and $\mathrm{Ni}(111)$.
\end{abstract}

\section{INTRODUCTION}

We have recently introduced a technique for probing local magnetic ordering using conventional angleresolved photoemission ${ }^{1-5}$ without spin detection of the photoelectrons. By measuring the $5 p$ core levels in different photoemission geometries, the magnetic ordering of $\mathrm{Gd}$ overlayers ${ }^{1-3}$ and $\mathrm{Tb}$ overlayers ${ }^{4,5}$ has been characterized. This technique is sensitive to the presence of conduction-band ferrimagnetic ordering, but does not distinguish the type of ferrimagnetic ordering. Nonetheless, we can probe the magnetic interactions between transition metals and rare earths by this technique, as has been described in preliminary communications. ${ }^{4,5}$

There has been considerable interest in the magnetic interactions between transition metals and rare earths of both an experimental ${ }^{4-21}$ and theoretical ${ }^{22-25}$ nature. While the majority of this work has been directed toward Gd overlayers ${ }^{6-11}, 14,15,17-20,22-25$ there is increasing interest in other rare-earth overlayers such as terbium. . $^{4,12,15-17}$ In general, it has been observed that the rare-earth overlayers couple antiferromagnetically with ferromagnetic substrates ${ }^{8-11,14,17}$ and exhibit properties (such as dichroism ${ }^{12}$ or Auger electron polarization ${ }^{15}$ ) attributable to magnetic ordering that are strongly dependent upon substrate temperature and overlayer thickness.

There has been an increasing effort to develop the Ginzburg-Landau equation to model the magnetization in overlayers deposited on ferromagnetic substrates. ${ }^{26-37}$ In this paper we demonstrate that for paramagnetic rare-earth overlayers, the changes in magnetization with changes in thickness are accurately described by a Ginzburg-Landau model. Terbium was chosen for these studies because of the low terbium Curie temperature $(215 \mathrm{~K})$ which permits investigation of the ferromagnetic and paramagnetic phases without complications associated with interdiffusion and alloy formation.

\section{EXPERIMENT}

The experiments were performed in an ultrahighvacuum (UHV) system equipped with a hemispherical analyzer for angle-resolved photoemission and a retarding-field analyzer for low-energy electron diffraction (LEED) described previously. ${ }^{3}$ The light source for the photoemission studies was the $1-\mathrm{GeV}$ ring at the Synchrotron Radiation Center, dispersed by 3-m and $6-\mathrm{m}$ toroidal grating monochromators. The energy resolution (analyzer and monochromator) of the photoemission spectra collected by the hemispherical analyzer varied from 0.15 to $0.3 \mathrm{eV}$ full width at half maximum.

The incidence angle of the light is defined with respect to the surface normal so that normal incidence (zero degrees) has vector potential completely parallel to the surface ( $s$-polarized) while glancing incidence light has a large component of the vector potential normal to the surface ( $p$ polarized) throughout this work to preserve symmetry selection rules.

Both the $\mathrm{Cu}(100)$ and $\mathrm{Ni}(111)$ surfaces were cleaned by $2-\mathrm{keV} \mathrm{Ar}^{+}$-ion bombardment and carefully annealed. Temperatures were determined by nickel-alumel thermocouples and the crystals could be cooled to $140-170 \mathrm{~K}$. Deposition of terbium followed procedures successfully developed for gadolinium as described elsewhere. ${ }^{3}$

The deposited thickness of our films was determined using an oscillating-crystal thin-film-thickness monitor, but the absolute thickness of our films was calibrated based upon changes in the core-level binding-energy shift and the relative terbium substrate photoemission signals. We have used substrate copper and terbium $4 f$ bindingenergy shifts to establish and confirm terbium coverages for coverages up to a monolayer. This procedure is based upon surface to bulk core-level shift arguments ${ }^{38,39}$ and has been successfully used with gadolinium overlayers. ${ }^{3,40}$

There is some evidence for copper interdiffusion with 
terbium overlayers even at room temperature. For this reason, results obtained in this work were repeated at substrate temperatures in the region of $200 \mathrm{~K}$. While interdiffusion of terbium with the nickel substrate cannot be completely ruled out, emission-angle-dependent studies suggest an abrupt interface and no evidence for interdiffusion was observed for substrate temperatures less than $300 \mathrm{~K}$.

As with gadolinium on $\mathrm{Cu}(100)$ (Ref. 3) and terbium on $\mathrm{Fe}(100),{ }^{15}$ the growth mode of terbium on both $\mathrm{Cu}(100)$ and $\mathrm{Ni}(111)$ is not epitaxial. We suggest that the growth mode is a simultaneous multilayer growth. For this reason, the thicknesses denote monolayer (ML) or angstrom equivalents and do not represent a uniform thickness, throughout this work.

\section{RESULTS}

There is a wealth of terbium valence features as seen in Figs. 1 and 2. This is, in part, a result of the $4 f$ multiplets. $^{41-44}$ The $4 f$ final states can be identified by comparison with $\mathrm{X}$-ray photoemission spectra. ${ }^{41-43}$ The various multiplets have binding energies at $2.2 \pm 0.1 \mathrm{eV}$ and $2.8 \pm 0.1 \mathrm{eV} \quad\left({ }^{8} S_{7 / 2}\right), \quad 7.6 \pm 0.1 \mathrm{eV}$ and $8.0 \pm 0.1 \mathrm{eV}$ $\left({ }^{6} \mathrm{D},{ }^{6} P,{ }^{6} I\right)$, and $9.5 \pm 0.1 \mathrm{eV}$ and $10.4 \pm 0.1 \mathrm{eV}\left({ }^{6} F,{ }^{6} H,{ }^{6} G\right)$ for thick films.

These terbium $4 f$ level binding energies are observed to change with overlayer thickness, particularly the ${ }^{8} S_{7 / 2}$ multiplet which overlaps the substrate $\mathrm{Cu} 3 d$ or $\mathrm{Ni} 3 d$ bands. The binding energies will also change with photon energy ${ }^{45}$ as a result of the changing oscillator strengths of the different $4 f$ levels. The feature at between $5.3 \pm 0.1 \mathrm{eV}$ to $5.9 \pm 0.1 \mathrm{eV}$ is an intrinsic feature that has been attributed to a many-electron correlation satellite described previously ${ }^{46}$ and is consistent with a re-

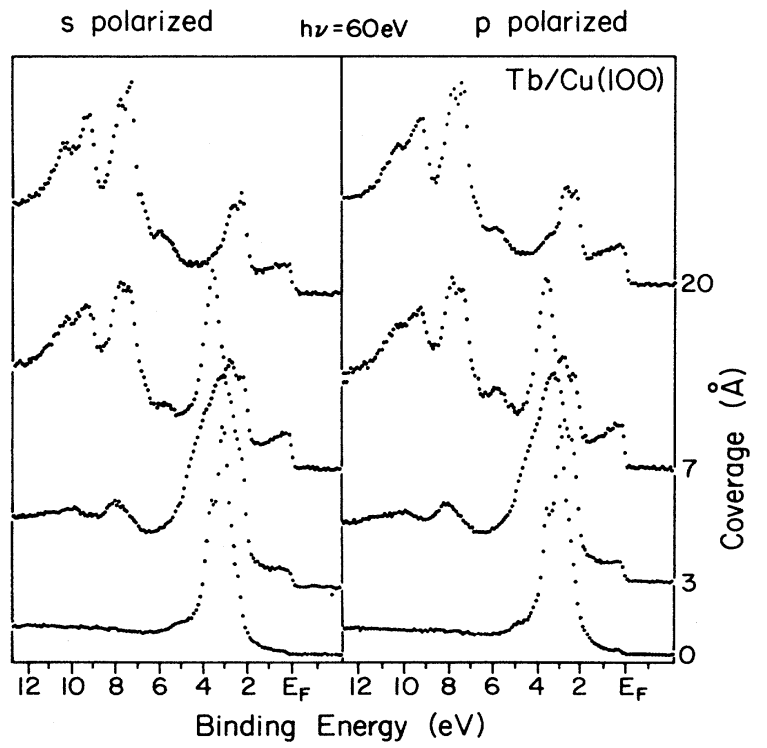

FIG. 1. Photoemission spectra of terbium overlayers on $\mathrm{Cu}(100)$, for light incidence angles of $34^{\circ}\left(s\right.$ polarized) and $70^{\circ}(p$ polarized). All photoelectrons were collected normal to the surface.

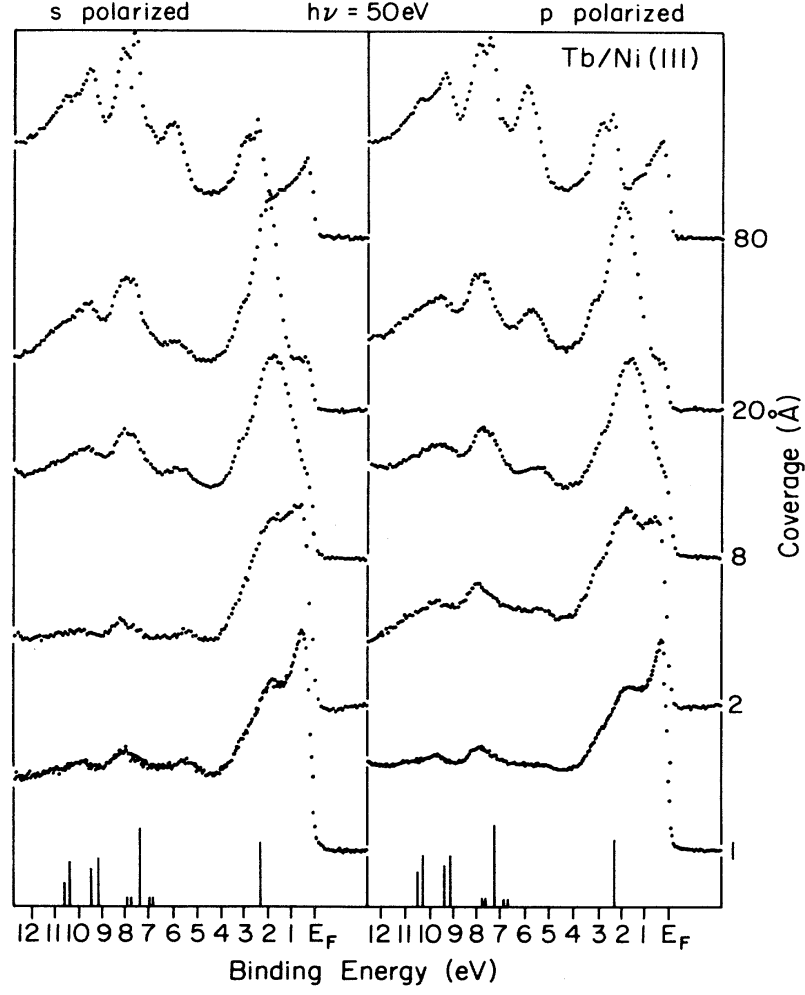

FIG. 2. Photoemission spectra of terbium overlayers on $\mathrm{Ni}(111)$, for light incidence angles of $40^{\circ}\left(\mathrm{s}\right.$ polarized) and $70^{\circ}(\mathrm{p}$ polarized). All photoelectrons were collected normal to the surface. The terbium $4 f$ multiplets are indicated at bottom.

cent experimental band structure of bulk terbium. ${ }^{47}$

For terbium on $\mathrm{Cu}(100)$, the terbium $5 d$ states have greater intensity with increasing $p$ polarization of the incident light, as seen in Fig. 1. This is readily apparent when comparing the $5 d$ intensity relative to the $\mathrm{Cu} 3 d$ states ( 3 to $3.8 \mathrm{eV}$ binding energy) and $\mathrm{Tb} 4 f$ multiplets. This is true, independent of coverage, except for terbium films of submonolayer coverage. Since the $4 f$ levels are less likely to have a well-defined spatial symmetry axis in one direction, ${ }^{3,43}$ from Fermi's golden rule we can infer that the terbium $5 d$ states are largely of $d_{3 z^{2}-r^{2}}$ character. Similar results have been obtained for gadolinium overlayers on $\mathrm{Cu}(100){ }^{3}$

Terbium overlayers on $\mathrm{Ni}(111)$ exhibit different $5 d$ symmetries for the states near the Fermi energy. There is a decreasing photoemission intensity of the combined $\mathrm{Ni}$ $3 d$ and $\mathrm{Tb} 5 d$ states at the Fermi energy (Fig. 2) with increasing $p$ polarization of the incident light for $1-2-\mathrm{ML}$ $\mathrm{Tb}$ films. This is most easily observed by comparing the $\mathrm{Tb} 5 d$ and $\mathrm{Ni} 3 d$ intensities with the $\mathrm{Tb} 4 f$ intensities. We can infer that the symmetry of these states is of largely $d_{x z, y z}$ character, since $s$-polarized light selectively excites these states. ${ }^{48,49}$ The $d$-band density of states away from the Fermi energy behaves in a reverse manner for thin [1-2 ML of terbium on $\mathrm{Ni(111)].} \mathrm{Increasing} p$ polarization of the incident light results in increased emission and these $d$-band states away from (not at $E_{F}$ ) the Fermi energy are therefore of $d_{3 z^{2}-r^{2}}$ character. As the 
thickness of the film increases, this behavior changes. For thick $\mathrm{Tb}$ films it is clear that the states at the Fermi energy are of $d_{3 z^{2}-r^{2}}$ character. A similar change in level ordering of the different symmetry bands with crystal substrate (as observed with terbium on copper as opposed to nickel) has been previously observed in halogen and chalcogen overlayers. ${ }^{50-52}$

The $\mathrm{Tb} 5 p_{1 / 2}$ and $5 p_{3 / 2}$ levels, at binding energies of $28.4 \pm 0.2 \mathrm{eV}$ and $22 . \pm 0.2 \mathrm{eV}$, respectively, also exhibit changing intensities with changing light polarization. Figure 3 shows these changes for terbium overlayers on $\mathrm{Ni}(111)$ and Fig. 4 terbium overlayers on $\mathrm{Cu}(100)$, and temperatures of about $170 \mathrm{~K}$.

The manner in which the terbium $5 p$ core levels depend on light polarization can be measured qualitatively on a relative scale from the ratio of $5 p_{3 / 2}$ to $5 p_{1 / 2}$ level intensities in different light polarization and normal emission. This ratio, the ratio of the $5 p$ branching ratios (the $5 p_{1 / 2}$ intensity divided by the $5 p_{3 / 2}$ intensity) for $p$ - and $s$-polarized light is denoted as the $p$-level anisotropy $\beta_{p} / \beta_{s} .{ }^{4}$ The $p$-level anisotropy has been measured and plotted for a variety of terbium film thickness on $\mathrm{Cu}(100)$ (Fig. 5) and $\mathrm{Ni}(111)$ (Fig. 6) well above (300 K) and well below $(170 \mathrm{~K})$ the Curie temperature of terbium $[215 \mathrm{~K}$ (Ref. 53)]. Note that for terbium on $\mathrm{Cu}(100)$, the $p$-level anisotropy measurements taken at room temperature are independent of coverage. These results shown in Fig. 5 demonstrate that the room-temperature $p$-level anisotropy of 0.82 is not the statistical value of 1.0. Above $T_{c}$, the $p$-level anisotropy depends on the overlayer coverage for terbium on $\mathrm{Ni}(111)$ (Fig. 6).

By changing the terbium overlayer temperature, there

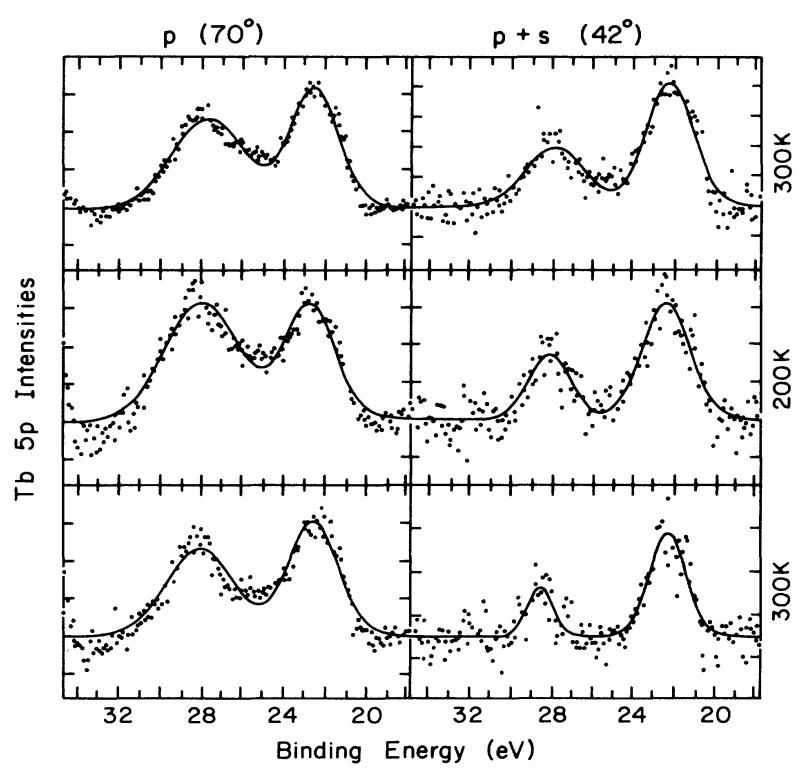

FIG. 3. The changing terbium $5 p$ intensities across the $\mathrm{Tb}$ Curie temperature for a one-monolayer (nominal film thickness) thick film of terbium on $\mathrm{Ni}(111)$. The changes are reversible. The photon energy is $60 \mathrm{eV}$, and all photoelectrons are collected normal to the surface..

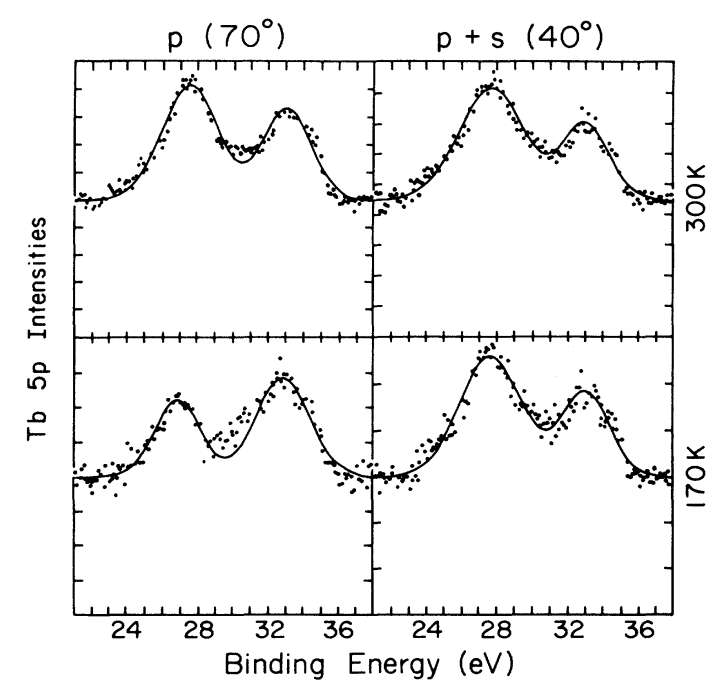

FIG. 4. The changing terbium $5 p$ intensities across the $\mathrm{Tb}$ Curie temperature for a monolayer (nominal film thickness) thick film of terbium on $\mathrm{Cu}(100)$. The photon energy is $60 \mathrm{eV}$, and all photoelectrons are collected normal to the surface.

are clearly significant changes in the $p$-level anisotropy as seen in Figs. 3 and 4 . These changes are reversible for several cycles of temperature change above and below $T_{c}$. For thin terbium overlayers on $\mathrm{Ni}(111)$, the $p$-level anisotropy is observed to increase with a decrease in temperature from above $T_{c}$ to below $T_{c}$. For the thick terbium films on $\mathrm{Ni}(111)$, the $p$-level anisotropy is observed to decrease from $1.6 \pm 0.2(300 \mathrm{~K})$ to $0.5 \pm 0.1(170 \mathrm{~K})$.

\section{DISCUSSION}

The $p$-level anisotropy is normally not expected to deviate from unity ${ }^{3}$ but as has been discussed in detail elsewhere ${ }^{1-4}$ some enhancement of the $5 p_{3 / 2}$ intensity is expected in some photoemission geometries as a result of long-range magnetic ordering. While core $p$ levels should

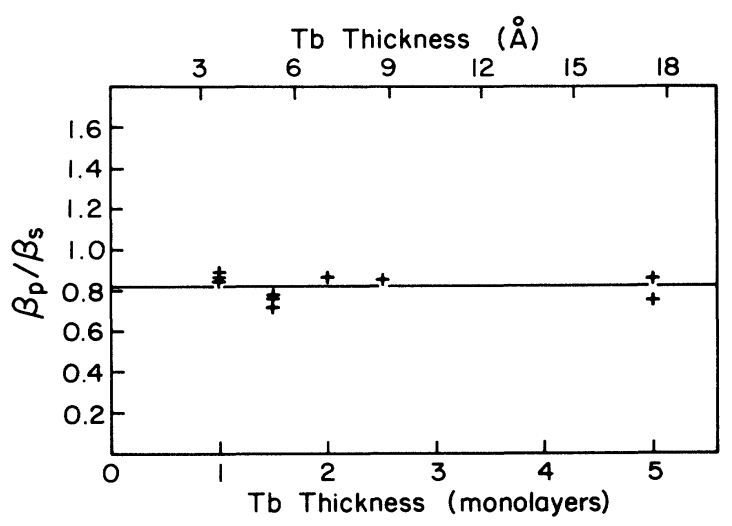

FIG. 5. The $p$-level anisotropy for terbium on $\mathrm{Cu}(100)$ at temperatures well above the terbium Curie temperature. Note that there is no variation with film thickness. Data are reduced from spectra as shown in Fig. 3. The line at 0.82 indicates the average value. 


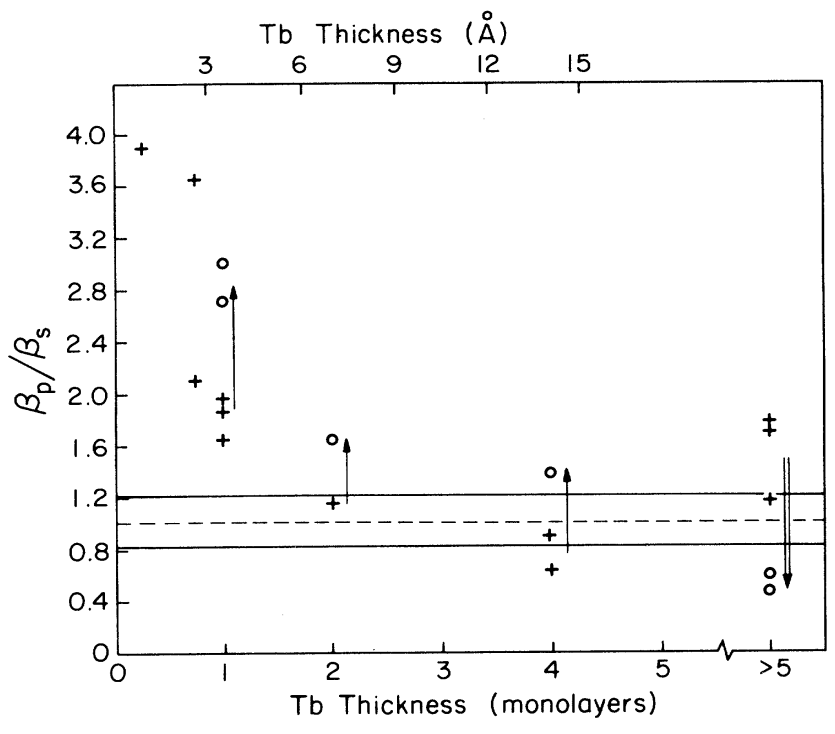

FIG. 6. The $p$-level anisotropy for terbium on $\mathrm{Ni}(111)$ at room temperature $(+)$ and at $170 \mathrm{~K}(0)$ as a function of film thickness. The expected $p$-level anisotropy of unity $(---)$ in the absence of ferromagnetic ordering is indicated. Crystal-field effect variations should fall between the two solid lines. The arrows indicate the reversible increase in the $p$-level anisotropy upon long-range ferromagnetic ordering. The double arrow indicates the change occurring with different $\left(d_{z^{3}-r_{2}}\right)$ symmetry at $E_{F}$ as discussed in the text.

not exhibit this behavior generally, because of final-state coupling of the $5 p$ photohole with the $5 d$ state, the outgoing electron can be of $d$ character and can exhibit some of the symmetry characteristics of the valence $5 d$ levels. ${ }^{3}$ This coupling can occur more strongly with the $5 p_{3 / 2}$ level than the $5 p_{1 / 2}$ because of the well-defined spin orientation of the initial-state eigenspinors that contribute to the $5 p_{3 / 2}$ level (states of $m_{j}= \pm \frac{3}{2}$ ). This coupling becomes anisotropic when there is some local spin ordering of the unoccupied $5 d$ states near the Fermi energy, 1,3,4 if we neglect crystal-field effects. If the terbium $5 d$ level does exhibit some magnetic ordering, enhancement of the $5 p_{3 / 2}$ feature over the $5 p_{1 / 2}$ level in some photoemission geometries should occur, consistent with our observations. Similar final-state photoemission effects have been observed by Fadley and co-workers ${ }^{54-57}$ for $3 d$ transition metals and have provided some measure of magnetic ordering. Related phenomena are also expected in $x$-ray absorption spectroscopy. ${ }^{58}$

Reversible changes in the $p$-level anisotropy, across the terbium Curie temperature $\left(T_{c}\right)$ cannot be a result of band structure or crystal field effects. Crystal-field effects should exhibit no temperature dependence. The results presented here indicate that the $p$-level anisotropy is a result, at least in part, of magnetic ordering as noted in a preliminary communication. ${ }^{4}$

Theoretical studies of rare-earth ${ }^{22,23}$ surfaces have suggested that the crystal-field potential at the surface may dominate and, as outlined by Goedkoop, ${ }^{12}$ could alter the $\mathrm{x}$-ray dichroism of terbium thin films. Arguments applied to gadolinium overlayers on $\mathrm{Cu}(100)$ (Ref. 1) and terbium overlayers on $\mathrm{Ni}(110)$ (Ref. 12) suggest that the crystal-field effects would have to be unrealistically large to account for unusual deviations in the $p$-level anisotropy from unity. While the $p$-level anisotropy may still, in part, be a result of crystal-field effects, the results presented here indicate that the $p$-level anisotropy is largely a result of magnetic ordering. The $p$-level anisotropies for terbium overlayers on $\mathrm{Cu}(100)$ deviate little from unity as seen in Fig. 5. Since the results in Fig. 5 are for terbium films at temperatures well above $T_{c}(300 \mathrm{~K})$ the results are representative of paramagnetic terbium. The average $p$-level anisotropy of $0.82 \pm 0.05$ differs by only $18 \%$ from unity. This deviation we attribute to crystal-field effects, or local magnetic ordering. Crystal-field effects are anticipated to be greater for the more open surfaces. ${ }^{12}$ Our crystal-field effect for $\mathrm{Cu}(100)$ is therefore likely to be larger than that observed for $\mathrm{Ni}(111)$.

While there are substantial $p$-level anisotropies observed above the terbium Curie temperature for thin terbium overlayers on $\mathrm{Ni}(111)$, this can be reconciled with magnetic ordering. At temperatures of $300 \mathrm{~K}$, while above the Curie temperature of terbium, this is well below the Curie temperature of nickel [627 K (Ref. 59)]. Thus, for very thin overlayers of terbium on $\mathrm{Ni}(111)$, the terbium overlayer is in the applied magnetic field of the substrate. This induces some magnetic ordering as in a paramagnet: ${ }^{59}$

$$
M=\mu_{B}^{2} B N\left(E_{F}\right) .
$$

This induced ordering will damp away from the interface. The damping will depend on a variety of factors such as the domain size, orientation of magnetization, and the coupling between terbium and nickel for ferromagnetic terbium, but for paramagnetic terbium, our results are consistent with the Ginzburg-Landau model of Mathon ${ }^{26-28}$ as we shall demonstrate below.

For thick films of terbium on $\mathrm{Ni}(111)$, the $p$-level anisotropy decreases dramatically away from unity as the temperature of the film is reduced to below $T_{c}$. Since the terbium $5 d$ band is of $d_{3 z^{2}-r^{2}}$ character for the thick films, we can compare our results with those obtained for gadolinium thin films on $\mathrm{Cu}(100)$ with identical $5 d$ character. For gadolinium on $\mathrm{Cu}(100)$, the $p$-level anisotropy was observed to decrease with decreasing temperature rather than increase, ${ }^{3}$ with some evidence suggesting that this occurred as a result of a component of magnetization along the surface normal. ${ }^{1}$ The results presented for ferromagnetic $\mathrm{Tb}$ on $\mathrm{Cu}(100)$ in Fig. 4 resemble those results reported for ferromagnetic $\mathrm{Gd}$ on $\mathrm{Cu}(100) .^{3}$ We conclude that the ferromagnetic ordering of thick terbium films is accompanied by a change in the $p$-level anisotropy. The $p$-level anisotropy serves as a means of probing and observing the onset and presence of ferromagnetic order in rare-earth containing films.

For the thinner films of terbium on Ni(111), the $p$-level anisotropy increases rather than decreases with increasing magnetic order (decreasing temperature) because the density of states near the Fermi energy has a $d_{x z, y z}$ like character, resulting in apparent changes in the $p$ to $d$ cou- 
pling in the final state. In general, our temperaturedependent results are consistent with temperature- and thickness-dependent dichroic effects observed for terbium overlayers on $\mathrm{Ni}(110)$ (Ref. 12) and polarization effects observed for terbium overlayers on $\mathrm{Fe}(100) .^{15}$

There is experimental evidence that the magnetization of the Ni(111) surface is in the plane of the surface. ${ }^{60}$ The results from the $p$-level anisotropy are consistent with this orientation of the magnetic moment because of the behavior of the $p$-level anisotropy which goes to unity as $z \rightarrow \infty$. The $p$-level anisotropies for $\mathrm{Tb}$ on $\mathrm{Ni}(111)$, for the thinner films, differ substantially from the $p$-level anisotropies observed for ferromagnetic $\mathbf{T b}$ and $\mathrm{Gd}$ on $\mathrm{Cu}(100)$ consistent with the different magnetization axis for these two systems obtained by combining $p$-level anisotropy and constant initial-state photoemission results. ${ }^{3}$ Large in-plane magnetizations have also been observed for $\mathrm{Gd}$ on $\mathrm{Fe}(100)$ (Refs. 14 and 15) and $\mathrm{Tb}$ on $\mathrm{Fe}(100),{ }^{15}$ for both $T>T_{c}$ and $T<T_{c}$. The substrate magnetization can overcome the tendency toward the anisotropic magnetization along the surface normal. The energy of the magnetic anisotropy is relatively small, even for the ferromagnet, with a value about $0.5 \mathrm{meV} /$ atom or less. ${ }^{61}$ Since the paramagnet will have a smaller anisotropy energy than the ferromagnet, it is unsurprising from Eq. (1) that the substrate magnetization can dominate the overlayers.

\section{GINZBURG-LANDAU THEORY APPLIED TO RARE-EARTH OVERLAYERS}

There have been a number of theoretical studies in which the spatially dependent magnetization $M(\mathbf{r})$ has been described for films, alloys, and ferromagnetic multilayers by applying the Ginzburg-Landau formulation. ${ }^{26-36}$ Here, we apply the method to a paramagnetic film on a ferromagnetic substrate and also make some comments on its extension to a ferromagnetic film. The free energy per unit volume in the Ginzburg-Landau formulation $^{35,62}$ is

$$
\begin{aligned}
f(r)= & f_{0}(r)+\frac{1}{2} A[\mathbf{M}(\mathbf{r})]^{2}+\frac{B}{4}[\mathbf{M}(\mathbf{r})]^{4}-\frac{1}{2} \mathbf{M}(\mathbf{r}) \cdot \mathbf{H}^{m} \\
& -\mathbf{M}(r) \cdot \mathbf{H}+\frac{1}{2} C[\nabla M(\mathbf{r})]^{2} .
\end{aligned}
$$

The first term $f_{0}(\mathbf{r})$ is the free energy per volume in the absence of magnetization. Also, the order parameter $M(\mathbf{r})$ is the magnetization in the film. If, for example, the substrate is a single domain ferromagnetic crystal, then the magnetization vector is $\mathbf{M}(\mathbf{r})=\widehat{\mathbf{e}}_{x} M(r)$, with the $x$ axis as the easy axis of magnetization. This assumes that the film is a linear magnetic material with magnetization parallel to the inducing field. The fields $\mathbf{H}^{m}$ and $\mathbf{H}$ are the demagnetization and applied field, respectively, while the Ginzburg-Landau coefficients $A, B$, and $C$ are temperature-dependent parameters. In particular, $A=A(T) \cong\left(T-T_{c}\right) \bar{\alpha}$ is generally assumed, with $\bar{\alpha}>0$ and independent of $T$. The constant $B$ is positive (or the transition would be first order) and can be related to spin and temperature. ${ }^{37,63}$ The temperature dependence of $A$ and $B$ near $T_{c}$ is rather suspect and does not agree with the available data. ${ }^{15,37}$ The constant $C$ is discussed by $\mathrm{Suhl}^{64}$ and Murata and Doniach ${ }^{65}$ and is positive. We will apply the formulation at a fixed temperature and thereby not require temperature dependence.

In the Gaussian approximation, the static magnetization $M_{0}(r)$ is found by solving $\delta F / \delta M=0$. This approximation will be valid except in the immediate vicinity of the critical point. Applying this, and setting $\mathbf{H}=\mathbf{0}$ yields $\mathbf{s}^{35,37,66}$

$$
A M_{0}(y)+B M_{0}(y)^{3}-C \frac{d^{2} M_{0}(y)}{d y^{2}}=H_{0}^{m}(y) .
$$

The coordinate $y$ is the distance of the considered plane in the overlayer film to the interface. Hence $0 \leq y \leq z$ where $z$ is the film thickness. From symmetry considerations, we expect $M_{0}(r)$ will not depend upon the in-plane coordinates. Thus $H_{0}^{m}(y)$ is the component of $\mathbf{H}^{m}$ along the magnetization direction.

Schwenk, Fishman, and Schwabl ${ }^{35}$ have considered the case of periodic multilayers of two alternating materials. When the in-plane dimensions are much greater than the layer thickness, they show that $H_{0}^{m}$ is zero. These conditions hold in our experiments, and we will neglect $H_{0}^{m}$. Both in our rare-earth experiments, and those of Landolt and co-workers, ${ }^{14,15}$ the magnetization appears to be largely "in plane." Thus in our experiment for paramagnetic $\mathrm{Tb}$ on $\mathrm{Ni}(111)$, those of Landolt and co-workers for $\mathrm{Tb}$ or $\mathrm{Gd}$ on $\mathrm{Fe}(100)$ (Refs. 14 and 15) and geometries of Schwenk and co-workers ${ }^{35} \mathbf{H}^{m}=\mathbf{B}-4 \pi \mathbf{M}$ and very restrictive boundary conditions apply, setting $\mathbf{H}^{m}=-4 \pi M_{\perp}=0$. Limiting ourselves to the static magnetization, and deleting the subscripts of Eq. (3), we can write

$$
-C \frac{d^{2} M(y)}{d y^{2}}+A M(y)+B[M(y)]^{3}=0 .
$$

Analysis of $M(\mathbf{r})$ as a scalar is valid (as indicated above) for the present results where $T>T_{c}$.

If $T<T_{c}$, a vector model is more appropriate as discussed elsewhere. ${ }^{65}$ Due to the antiferromagnetic coupling of the rare-earth overlayers with transition-metal substrates, ${ }^{11,15,17}$ both parallel and perpendicular components of $\mathbf{M}(\mathbf{r})$ (Ref. 12) exist and must be considered. A parallel component arises from the necessity of satisfying $\boldsymbol{\nabla} \cdot \mathbf{B}=0$ and the shape anisotropy.

For $T<T_{c}$ we can write $\mathbf{M}(\mathbf{r})=M_{\|}(\mathbf{r}) \widehat{e}_{\|}+M_{\perp}(\mathbf{r}) \widehat{e}_{\perp}$, with $M_{\|}$and $M_{\perp}$ as the components of $\mathbf{M}$ that are parallel and perpendicular to the interface, respectively. Noting that the third term of Eq. (2) is $(B / 4)[\mathbf{M}(\mathbf{r}) \cdot \mathbf{M}(\mathbf{r})]^{2}$, Eq. (2) becomes

$$
\begin{aligned}
f(\boldsymbol{r})= & f_{0}(r)+\frac{A}{2} M_{\|}(\mathbf{r})^{2}+\frac{A}{2} M_{\perp}(r)^{2}+\frac{B}{4} M_{\|}(\mathbf{r})^{4} \\
& +\frac{B}{2} M_{\|}(\mathbf{r})^{2} M_{\perp}(\mathbf{r})^{2}+\frac{B}{4} M_{\perp}(r)^{4}-\frac{1}{2} \mathbf{M} \cdot \mathbf{H}^{m}-\bar{M} \cdot \mathbf{H} \\
& +\frac{C}{2}\left[\nabla M_{\|}(\mathbf{r})\right]^{2}+\frac{C}{2}\left[\nabla M_{\perp}(\mathbf{r})\right]^{2}
\end{aligned}
$$

The condition $\delta F / \delta M=0$ is replaced by $\delta F / \delta M_{\|}=0=\delta F / \delta M_{\perp}$ to yield a pair of differential equations for $M_{\|}$and $M_{\perp}$ that replaces Eq. (3). Due to 
the term $(B / 2) M_{\perp}(\mathbf{r})^{2} M_{\|}(\mathbf{r})^{2}$, these two equations will be coupled. These two coupled equations are then

$$
\begin{aligned}
& -C \frac{d^{2} M_{\|}(y)}{d y^{2}}+A M_{\|}(y)+B M_{\|}(y) M(y)^{2}=H_{\|}^{m}, \\
& -C \frac{d^{2} M_{\perp}(y)}{d y^{2}}+A M_{\perp}(y)+B M_{\perp}(y) M(y)^{2}=H_{\perp}^{m},
\end{aligned}
$$

where, under some circumstances (i.e., very thin films), $H_{\|}^{m}=0 .{ }^{35}$

Mathon has argued ${ }^{26}$ that the Ginzburg-Landau theory can be applied to palladium overlayers on nickel for thickness values less than 10 to 15 monolayers. Here we apply the theory to results obtained for terbium films of thicknesses five monolayers or less.

The demagnetization field $H^{m}$ is negligible in our paramagnetic overlayer. Returning to $T>T_{c}$, setting $\mathbf{H}^{m}$ to zero as in Eq. (4), and integrating Eq. (4), yields

$$
\frac{1}{\kappa^{2}}\left[\frac{d M(y)}{d y}\right]^{2}=[M(y)]^{2}+b[M(y)]^{4}+a,
$$

where $b=B / 2 A$ and $a$ is a constant of integration. The correlation length, dependent upon bulk properties of the overlayer, is $\kappa^{-1}$ and is related to short-range magnetic order. ${ }^{37}$ The value $\kappa$ is defined by $\kappa=(A / C)^{1 / 2}$.

The second integral yields the solution for $M(y)$ :

$$
\kappa y=\int_{M(y)}^{M(0)} \frac{d M(y)}{\left\{b[M(y)]^{4}+[M(y)]^{2}+a\right\}^{1 / 2}}
$$

assuming that $M(0)>M(y)$ within the layer, as is certainly the case for a paramagnetic layer on a ferromagnetic substrate. We may also expect the sign choice for the square root to be positive for the case of a ferromagnetic layer on a ferromagnetic substrate if the substrate is the stronger ferromagnet.

The two constants of integration of Eq. (6), $M(0)$ and $a$, can be conveniently expressed using length parameters $\lambda$ and $\gamma$ by

$$
\begin{aligned}
& \frac{1}{\gamma}=\left.\frac{-1}{M(0)} \frac{d M(y)}{d y}\right|_{y=0}, \\
& \frac{1}{\lambda}=\left.\frac{-1}{M(z)} \frac{d M(y)}{d y}\right|_{y=z} .
\end{aligned}
$$

The length $\lambda$ describes the free surface and is analogous to the free surface extrapolation length introduced by Binder and Hohenberg ${ }^{29}$ in their discussion of the Lenz-Ising model. Similarly, $\gamma$ characterizes the interface. Mathon ${ }^{26,27}$ has given values for $\lambda$ and $\gamma$ by studying magnetization data for palladium films on a nickel substrate.

Combining Eqs. (5) and (7) yields a pair of equations relating the five constants $a, M(0), M(z), \lambda$, and $\gamma$ :

$$
\begin{aligned}
& {\left[(\kappa \gamma)^{-2}-1\right][M(0)]^{2}-b[M(0)]^{4}=a,} \\
& {\left[(\kappa \gamma)^{-2}-1\right][M(z)]^{2}-b[M(z)]^{4}=a .}
\end{aligned}
$$

By substituting $z=y$ into Eq. (6) we can obtain

$$
\kappa z=\int_{M(z)}^{M(0)} \frac{d M(y)}{\left\{b[M(y)]^{4}+[M(y)]^{2}+a\right\}^{1 / 2}} .
$$

Hence if any two of the five constants are known, the remaining constants can be ascertained by applying Eqs. (8) and (9).

Interpretation of $\lambda$ follows from Eq. (7b). If $M(y) \sim e^{-\kappa y}$ describes the magnetization near the free surface, then $\kappa \lambda=1$. If the free surface magnetization is larger than predicted by an exponential decay (a large penetration depth $\kappa^{-1}$ ) then $\kappa \lambda>1$, corresponding to a small value for $\left|M^{\prime}(y)\right|_{y=z}$. However, $M(z)<M(y)$ will still hold for $y<z$, thus the free surface is weakly enhanced. When $0<\kappa \lambda<1$, then $\left|M^{\prime}(y)\right|_{y=z}$ is of a larger size than would be the case for a simple exponential decay. Then the surface magnetization is suppressed. If $-1<\kappa \lambda<0$, then there is an increase in $M(z)$, relative to the layers beneath the surface. In this case $\left.M^{\prime}(y)\right|_{y=z}$ is positive and large (thus representing a live surface layer). Finally, for $\kappa \lambda<-1, M(y)$ increases more strongly than $\exp (\kappa y)$ near the free surface and the surface layer is strongly enhanced. It should be noted that if $M(y)$ obeys $M(y)=M(0) e^{-\kappa y}$ throughout the entire film, then $\lambda=\gamma=\kappa^{-1}$.

The integral in Eq. (6) can be cast in the form of incomplete elliptic integrals of the first kind defined by

$$
F(\phi, k)=\int_{0}^{\phi} \frac{d \alpha}{\left(1-k^{2} \alpha^{2}\right)^{1 / 2}} .
$$

As noted already, $B>0$ and hence $b>0$ for paramagnetic terbium. Standard identities ${ }^{67}$ allow the solutions for $M(y)$ to be rewritten. For the case $0<4 a b<1$,

$$
M_{+} \sqrt{b} \kappa y=F\left(\alpha_{0}, q\right)-F\left(\alpha_{y}, q\right)
$$

with

$$
\begin{aligned}
& M_{ \pm}=(2 b)^{-1 / 2}(1 \pm \sqrt{1-4 a b}) \\
& q=\left(M_{+}^{2}-M_{-}^{2}\right)^{1 / 2} / M_{+} \\
& \alpha_{y}=\arctan \left[M(y) / M_{-}\right] .
\end{aligned}
$$

If $4 a b>1$, then

$$
(a b)^{1 / 4} \kappa y=F\left(\beta_{y}, \xi\right)-F\left(\beta_{0}, \xi\right),
$$

with

$$
\begin{aligned}
& \cos \beta_{y}=\frac{M(y)^{2}-\sqrt{a / b}}{M(y)^{2}+\sqrt{a / b}}, \\
& \xi=\frac{1}{2}(a b)^{-1 / 4}\left[\left(1+\eta^{2}\right)^{1 / 2}-1\right], \\
& \eta=4 a b-1 .
\end{aligned}
$$

For $a<0$, the result is

$$
\sqrt{\varphi} \kappa y=F\left(\epsilon_{0}, S\right)-F\left(\epsilon_{y}, S\right),
$$

with

$$
\begin{aligned}
& \varphi=\sqrt{1+4|a| b} \\
& \cos \epsilon_{y}=\frac{1}{M(y)} \sqrt{(\varphi-1) / 2 b}
\end{aligned}
$$




$$
S=\frac{1}{\sqrt{2}} \sqrt{(1 / \varphi)+1}
$$

In principle, if sufficiently extensive data for $M(y)$ are available, comparison with Eqs. (11), (13), and (15) will allow determination of $\kappa, b, a$, and $M(0)$. This would permit us to derive $\lambda$ and $\gamma$. Here, however, we will only attempt to fit our data to the case of $b=0$, in harmony with the fact that $b$ is generally very small for a paramagnetic material. The result for $M(y)$ can be found from Eq. (4) or from Eq. (6) by setting $b=0$. The result is

$$
M(y)=R e^{-\kappa y}+T e^{\kappa y},
$$

where the two constants of integration, $R$ and $T$, replace the original pair of constants $M(0)$ and $a$, and obey $R>T>0$.

The function $M(y)$ is really a function of $y$ and $z$, i.e., $M(y, z)$. Since photoemission is a surface sensitive probe under our experimental conditions we have, in fact, measured $M(z, z)$. Assuming $M(y, z)$ is independent of $z$, we then write

$$
M(z)=R e^{-\kappa z}+T e^{\kappa z},
$$

which corresponds to our measurement.

Since for very thick films, the free surface magnetization approaches zero, the constant $T \rightarrow 0$. This implies $T$ is small. In fitting Eq. (17) to the data for paramagnetic terbium, we restrict the fit to the case for which $T<R$, allowing for the second term in Eq. (17) to be omitted. Thus

$$
M(z) \cong R e^{-\kappa z} .
$$

The $p$-level anisotropy is unity with no magnetic ordering and (neglecting crystal-field effects) thus

$$
\frac{\beta_{p}(z)}{\beta_{s}(z)}-q=p M(z),
$$

where $p$ is an appropriate scaling constant, and $q$ is unity with no crystal-field effects (and 0.82 to 1.2 using our experimentally measured crystal-field effect and assuming this deviation from unity is not altered by short-range magnetic order). We have fit this equation to our data for terbium on $\mathrm{Ni}(111)$ as shown in Fig. 7 (assuming $q \simeq 1.0$ ), and the agreement with this exponential profile is good. In fitting this data, we use the fact that photoemission is surface sensitive. The small deviations of the experimental data from the exponential profile are a result of errors in our estimation of the terbium coverage.

This Ginzburg-Landau theory can also be applied to the results obtained for terbium on $\mathrm{Fe}(100)$ from Auger electron polarization studies by Paul, Landolt, and coworkers. ${ }^{15}$ These results plotted in Fig. 8 have been fitted to Eq. (18) as well. For terbium on $\mathrm{Ni}(111)$ we ascertain that the correlation length $\kappa^{-1}=0.71 \pm 0.19$ monolayers $(2.5 \pm 0.7 \AA)$ which is in reasonable agreement with the value of $\kappa^{-1}=1.0$ monolayers $(\sim 3.5 \AA)$ obtained for terbium on $\mathrm{Fe}(100)$ considering the difficulties in estimating the terbium overlayer thickness and the differences in the electron mean free path (the surface sensitivity) between the two spectroscopies employed. (Corrections for the

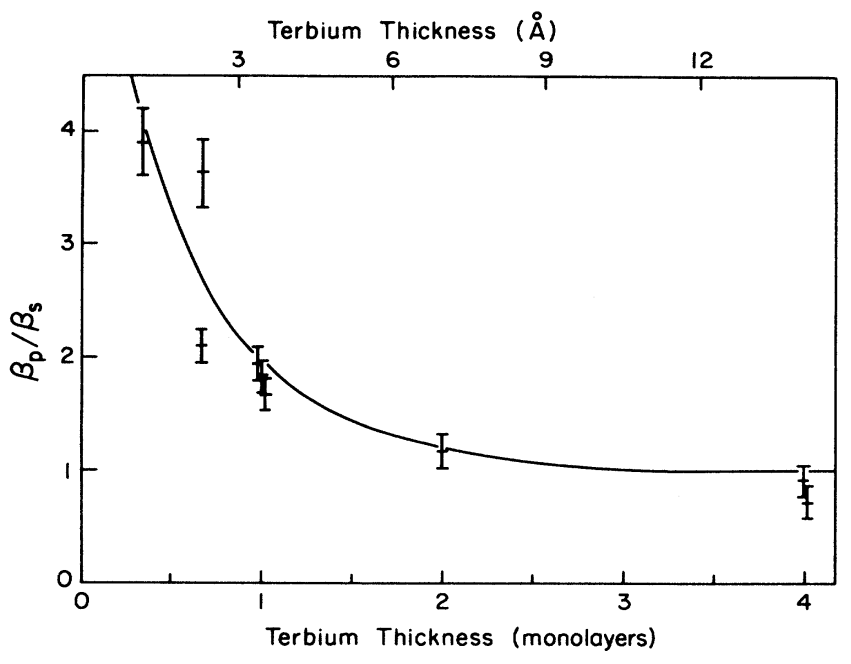

FIG. 7. The Ginzburg-Landau model fitted to the $p$-level anisotropy data for paramagnetic terbium. The values of $\kappa^{-1}=0.71(\sim 2.5 \AA)$ and $q=1$ have been used.

experimentally derived crystal-field effects have little effect on $\kappa^{-1}$.)

As pointed out earlier, the correlation length is related to the short-range magnetic order for $T>T_{c}$ and is, in principle, a bulk property. ${ }^{37,66}$ For bulk terbium the inverse correlation length has been obtained from the short-range magnetic order measured by neutron diffraction. ${ }^{68}$ Extrapolating these results to our temperature of $300 \mathrm{~K}$ gives us values of $\kappa^{-1}=2.8 \AA$ along the $c$ axis and $\kappa^{-1}=4.9 \AA$ along the basal plane of bulk terbi-

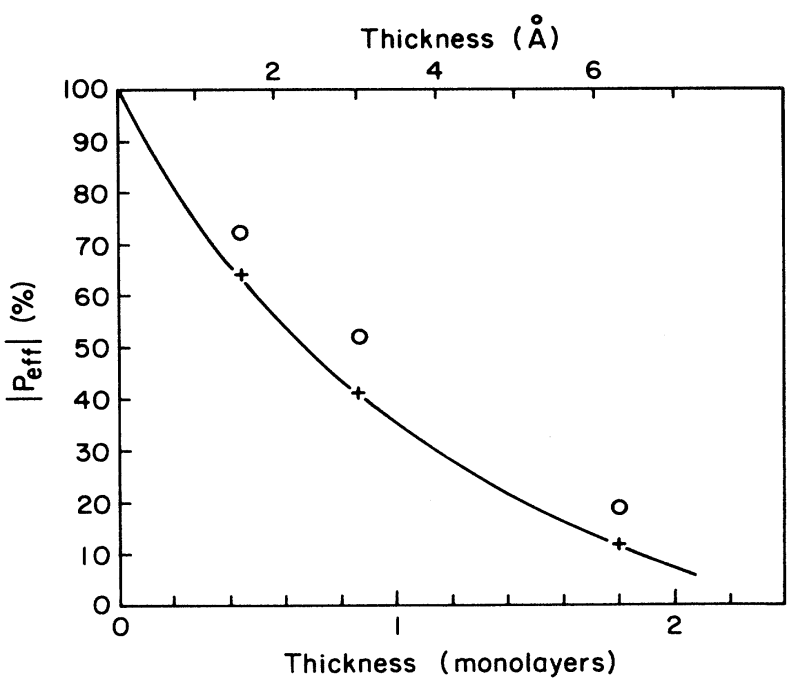

FIG. 8. The Ginzburg-Landu model fitted to the Auger electron polarization data for terbium on $\mathrm{Fe}(100)$ taken from Ref. 15. Data for room temperature $(+)$ and $170 \mathrm{~K}(O)$ have been plotted. The Ginzburg-Landau model has been fitted to the room-temperature data using a value of $\kappa^{-1}=0.98(\sim 3.5 \AA)$. Notice the overall similarity of this data and those shown in Fig. 6. 
um. The correlation length for bulk terbium along the $c$ axis is in surprisingly good agreement with our value of $\kappa^{-1}$. This result suggests that the terbium overlayer, while showing no crystal order in LEED or reflection high-energy electron diffraction (RHEED), grows with an hcp structure with the basal plane parallel with the surface, or least has a $\mathrm{Tb}$ - $\mathrm{Tb}$ lattice constant close to that of the in-plane basal face lattice constant.

The effective values obtained for the correlation length $\kappa^{-1}$ for paramagnetic terbium overlayers $(2.5-3.5 \AA$ or less than an atomic diameter) are much less than the correlation length of $\kappa^{-1}=5 \AA$ (much more than an atomic diameter of $\mathrm{Pd}$ of $2.75 \AA$ ) obtained for palladium (a nearly ferromagnetic metal) on nickel. ${ }^{27,31}$ This difference is particularly apparent if one considers that the experimental spectroscopies employed are not perfectly surface sensitive. This is unsurprising in view of the far more localized interactions expected for the rareearth metals as opposed to the transition metals. The terbium results are in good agreement with the correlation length of $2.5 \pm 0.5 \AA$ estimated for $\mathrm{Gd}$ on $\mathrm{Fe}(100)$ at $T / T_{c}=1.22$ (Ref. 14) (as compared with a $T / T_{c}=1.4$ for the terbium results reported herein).

Recall, if the decay rate is a simple exponential $M(y) \sim e^{-\kappa y}$ over $0 \leq y \leq z$, then $\lambda^{-1}=\kappa$ follows from Eq. (7). Returning to the data for $\mathrm{Tb}$, the fact that its paramagnetic phase follows an exponential decay so well indicates that $\kappa \lambda \sim 1$. Hence there exists at most very weak suppression or very weak enhancement at the $\mathrm{Tb}$ free surface. The $T b$ free surface acts like a paramagnet and does not show either strong enhancement or "live" behavior. A similar result (i.e., $\kappa \lambda \sim 1$ ) was found by Gradmann and Bergholz ${ }^{62}$ for Pd overlayers on $\mathrm{Ni}$, at room temperature.

Boundary conditions [Eqs. (7) and (8)] at the interface result in a diminution of the magnetization at the ferromagnetic substrate surface as a result of the paramagnetic overlayer. Spin-dependent photoemission studies of terbium on $\mathrm{Fe}(100)$ (Ref. 17) have observed a reduction of the polarization of the $\mathrm{Fe}(100)$ substrate valence emission, consistent with these boundary conditions.

\section{CONCLUSION}

By measuring the $p$-level anisotropy above and below the terbium Curie temperature, we have demonstrated conclusively that the $p$-level anisotropy can be used to probe magnetic order in rare-earth overlayers. Our results show that terbium overlayers magnetically interact with ferromagnetic substrates such as nickel or iron. The ferromagnetic substrates clearly induce some magnetic order in very thin $(<4$ monolayer equivalents) terbium films which damped with increasing distance away from the interface in a manner consistent with GinzburgLandau theory. We find the correlation length for terbium to be $0.71 \leq \kappa^{-1} \leq 1.0$ monolayer $(2.5-3.5 \AA)$ at 300 $\mathrm{K}$. This result is consistent with the bulk correlation length, and results reported for gadolinium. ${ }^{14}$

The smaller correlation length found for paramagnetic terbium, in comparison with paramagnetic $\mathrm{Pd}$ may reflect that the magnetic interactions in the rare-earth metals are far more localized than in palladium. Our experimental and theoretical analysis indicates that paramagnetic terbium on $\mathrm{Ni}(111)$ exhibits a magnetization that follows an exponential decay from the ferromagnet-paramagnet interface.

We have also established that paramagnetic terbium on $\mathrm{Fe}(100)$ exhibits an induced magnetization that decays exponentially away from the interface using the results of Landolt and co-workers. ${ }^{15}$ It is unsurprising that both our $p$-level anisotropy data and Landolt and co-workers' effective electron spin-polarization data lead to the same conclusion, given the results of the Ginzburg-Landau theory.

\section{ACKNOWLEDGMENTS}

This work was supported by the U.S. Department of Energy through Grant No. DE-FG-02-87-ER45319. The work was undertaken at the Synchrotron Radiation Center (SRC) in Stoughton, Wisconsin. The SRC is funded by the National Science Foundation. The authors would like to thank John Fuggle for sharing the results of his work and that of his student, J. Goedkoop, and M. Landolt for critically reading the manuscript.
*To whom all correspondence may be addressed.

${ }^{1}$ D. LaGraffe, P. A. Dowben, and M. Onellion, Phys. Rev. B 40, 970 (1989).

${ }^{2}$ D. LaGraffe, P. A. Dowben, and M. Onellion, Mater. Res. Soc. Symp. Proc. 151, 71 (1989).

${ }^{3}$ P. A. Dowben, D. LaGraffe, and M. Onellion, J. Phys. Condens. Matter 1, 6751 (1989).

${ }^{4}$ D. LaGraffe, P. A. Dowben, and M. Onellion, Phys. Lett. A 147, 240 (1990).

${ }^{5}$ D. Lagraffe, P. A. Dowben, A. Miller, and M. Onellion, Mater. Res. Soc. Symp. Proc. 187, (1991).

${ }^{6}$ Y. Kamiguchi, Y. Hayakawa, and H. Fulimore, Appl. Phys. Lett. 55, 1918 (1989).

${ }^{7}$ T. Morishita, Y. Togami, and K. Tsuchima, J. Phys. Soc. Jpn. 54, 37 (1985).

${ }^{8}$ D. Mauri and M. Landolt, Phys. Rev. Lett. 47, 1322 (1981).

${ }^{9}$ A. Cerri, D. Mauri, and M. Landolt, Phys. Rev. B 27, 6526 (1983).

${ }^{10}$ M. Landolt, R. Allenspach, and M. Taborelli, Surf. Sci. 179,
311 (1986).

${ }^{11}$ C. Carbone and E. Kisker, Phys. Rev. B 36, 1280 (1987).

${ }^{12}$ J. Goedkoop, Ph. D. dissertation, Katholieke Universiteit te Nijmegen (1989).

${ }^{13}$ Teruya Shinjo, K. Mibu, S. Ogawa, and N. Hosoito, Mater. Res. Soc. Symp. Proc. 151, 87 (1989).

${ }^{14}$ M. Taborelli, R. Allenspach, G. Boffa, and M. Landolt, Phys. Rev. Lett. 56, 2869 (1986).

${ }^{15}$ O. Paul, S. Toscano, W. Hürsch, and M. Landolt, J. Magn. Magn. Mater. 84, L7 (1990).

${ }^{16}$ C. Rau, C. Jin, and G. Xing, Phys. Lett. A 144, 406 (1990).

${ }^{17}$ C. Carbone, R. Rochow, L. Braicovich, R. Jungblut, T. Kachel, D. Tillman, and E. Kisker, Phys. Rev. B 41, 3866 (1990).

${ }^{18}$ Z. S. Shan, D. J. Sellmyer, S. S. Jaswal, Y. J. Wang, and J. X. Shen, Phys. Rev. Lett. 63, 449 (1989).

${ }^{19}$ B. Dieny, D. Givord, J. M. B. Ndjaka, and J. M. Alameda, J. Appl. Phys. 69, 5677 (1990).

${ }^{20}$ G. Choe, A. P. Valanju, and R. M. Walser, J. Appl. Phys. 67, 5671 (1990). 
${ }^{21}$ Z. S. Shan and D. J. Sellmyer, J. Appl. Phys. 67, 5713 (1990).

${ }^{22}$ I. Peschel and P. Fulde, Z. Phys. 259, 145 (1973).

${ }^{23}$ Y. Y. Hsie and D. A. Pink, J. Phys. Chem. Solids 35, 1481 (1974).

${ }^{24}$ R. E. Camley, Phys. Rev. B 35, 3608 (1987).

${ }^{25}$ S. Blugel, H. Akai, R. Zeller, and P. H. Dederichs, Phys. Rev. B 35, 3271 (1987).

26J. Mathon, J. Phys. F 16, L217 (1986).

${ }^{27}$ J. Mathon, J. Phys. F 16, 669 (1986).

${ }^{28}$ J. Mathon and G. Bergmann, J. Phys. F 16, 887 (1986).

${ }^{29}$ K. Binder and P. C. Hohenberg, Phys. Rev. B 6, 3461 (1972).

${ }^{30}$ D. M. Edwards, J. Mathon, and E. P. Wohlfarth, J. Phys. F 3, 161 (1973).

${ }^{31}$ D. M. Edwards, J. Mathon, and E. P. Wohlfarth, J. Phys. F 5, 1619 (1975).

${ }^{32}$ S. Coutinho, D. M. Edwards, and J. Mathon, J. Phys. F 13, L143 (1983).

${ }^{33}$ D. R. Tilley, Solid State Commun. 65, xxx (1988).

${ }^{34}$ R. E. Camley, Phys. Rev. B 35, 3608 (1987).

${ }^{35}$ F. Fishman, F. Schwabl, and D. Schwenk, Phys. Lett. A 121, 192 (1987); D. Schwenk, F. Fishman, and F. Schwabl, Phys. Rev. B 38, 11618 (1988).

${ }^{36}$ J. A. Tuszynski, Mater. Res. Soc. Symp. Proc. 151, 265 (1989).

${ }^{37}$ C. Domb and M. S. Green, Phase Transitions and Critical Phenomena (Academic, New York, 1976), Vol. 5A.

${ }^{38}$ W. F. Egelhoff, Jr., Surf. Sci. Rep. 6, 253 (1987); Phys. Rev. B 29, 4769 (1984); Phys. Rev. Lett. 50, 587 (1983).

${ }^{39}$ M. Salmeron, S. Ferrer, M. Jazzar, and G. A. Somorjai, Phys. Rev. B 28, 6758 (1983); 28, 1158 (1983).

${ }^{40}$ D. LaGraffe, P. A. Dowben, and M. Onellion, Phys. Rev. B 40, 3348 (1989).

${ }^{41}$ Y. Baer and G. Busch, J. Electron Spectrosc. Relat. Phenom. 5, 611 (1974).

42 J. K. Lang, Y. Baer, and P. A. Cox, J. Phys. F 11, 121 (1981).

${ }^{43}$ P. A. Cox, J. K. Lang, and Y. Baer, J. Phys. F 11, 113 (1981).

${ }^{44}$ P. A. Cox, in Structure and Bonding, Vol. 24 of Springer Verlag Tracts, edited by EDITORS (Springer-Verlag, New York, 1974), p. 59.

${ }^{45}$ P. A. Dowben, D. LaGraffe, Dongqi Li, L. Dottl, Y. Ufukteppe, C. Hwang, and M. Onellion, J. Phys. Condens. Matter 2, 8801 (1990).

${ }^{46}$ D. LaGraffe, P. A. Dowben, and M. Onellion, J. Vac. Sci. Technol. A 8, 2738 (1990).

${ }^{47}$ S. C. Wu, H. Li, D. Tian, J. Quinn, Y. S. Li, F. Jona, J. Sokolov, and N. E. Christensen, Phys. Rev. B 41, 11911 (1990).

48 J. Hermanson, Solid State Commun. 22, 9 (1977).

${ }^{49}$ W. Eberhardt and F. J. Himpsel, Phys. Rev. B 21, 5572 (1980).

${ }^{50}$ K. Jacobi, M. Scheffler, K. Kambe, and F. Forstman, Solid
State Commun. 22, 17 (1977).

${ }^{51}$ W. Plummer, B. Tonner, N. Holtzworth, and A. Liebsch, Phys. Rev B 21, 4306 (1980).

${ }^{52}$ P. A. Dowben, CRC Reviews in Solid State and Materials Sciences (Chemical Rubber, Cleveland, 1987), Vol. 13, p. 195, and the references therein.

${ }^{53} \mathrm{~K}$. A. McEwen, in Handbook of the Physics and Chemistry of Rare Earths, edited by K. A. Gschneider and L. Eyring (North-Holland, Amsterdam, 1982).

${ }^{54}$ B. Hermsmeier, J. Osterwalder, D. J. Friedman, and C. S. Fadley, Phys. Rev. Lett. 62, 478 (1989).

${ }^{55}$ B. Sinković, B. Hermsmeier, and C. S. Fadley, Phys. Rev. Lett. 55, 1227 (1985).

${ }^{56}$ B. Sinković and C. S. Fadley, Phys. Rev. B 31, 4665 (1985).

${ }^{57}$ C. S. Fadley, in Magnetic Properties of Low Dimensional Systems II, Vol. 50 of Springer Proceedings in Physics, edited by L. M. Falicov, F. Mejia-Lira, and J. L. Moran-Lopez (Springer-Verlag, Berlin, 1990), p. 36; Phys. Scr. T 17, 39 (1987).

${ }^{58}$ G. van der Laan and B. T. Thole, Phys. Rev. Lett. 60, 1977 (1988).

${ }^{59}$ N. W. Ashcroft and N. D. Mermin, Solid State Physics (Saunders College, Philadelphia, 1976), see p. 663.

${ }^{60} \mathrm{For}$ both $\mathrm{Fe}(100)$ and $\mathrm{Ni}(111)$, these surfaces also represent the "hard" (-magnetization) directions while the other low-index faces are also directions that are "easy," see C. Kittel, Introduction to Solid State Physics, 5th ed. (Wiley, New York, 1976), p. 487; C. A. Ballentine, R. L. Fink, J. Araya-Pochet, and J. L. Erskine, Phys. Rev. B 41, 2631 (1990).

${ }^{61}$ J. G. Gay and R. Richter, Phys. Rev. Lett. 56, 2728 (1986).

${ }^{62} \mathrm{U}$. Gradmann and R. Bergholz, Phys. Rev. Lett. 52, 771 (1984).

${ }^{63}$ D. L. Mills, Phys. Rev. B 3, 3887 (1971).

${ }^{64}$ H. Suhl, Appl. Phys. 8, 217 (1975).

${ }^{65}$ K. K. Murata and S. Doniach, Phys. Rev. Lett. 29, 285 (1972).

${ }^{66}$ E. M. Lifshitz and L. P. Pitaevski, Statistical Physics, Part 2 (Pergamon, Oxford, 1980), p. 182.

${ }^{67}$ I. S. Grodskteyn and I. M. Ryzhik, Tables of Integrals, Series and Products, corrected and enlarged edition (Academic, New York, 1985); see p. 245.

${ }^{68}$ The inverse correlation length is taken from neutronscattering data taken near $T_{c}$, so our extrapolation to $300 \mathrm{~K}$ may not be completely valid. The original data come from $\mathrm{O}$. W. Dietrich and J. Als-Nielsen, Neutron Inelastic Scattering, Proceedings of a Symposium (IAEA, Vienna, 1968), Vol. II, pp. 63-73 and O. W. Dietrich and J. Als-Nielsen, J. Phys. C 4, 71 (1971), and the data are also presented in Ref. 37 on p. 147. 